\title{
Improvement of Dissolution Rate of Gliclazide Through Sodium Salt Formation
}

\author{
Dina El-Sabawi* and Imad I. Hamdan \\ Faculty of Pharmacy, The University of Jordan, Amman 11942, Jordan
}

\begin{abstract}
Gliclazide is a hypoglycemic agent exhibiting to some extent inadequate and variable absorption as a consequence of poor aqueous solubility and slow dissolution rates. A sodium salt of gliclazide was prepared and investigated for solubility and dissolution properties in comparison to untreated gliclazide. The salt was formed by adding equimolar amounts of gliclazide and sodium hydroxide in an aqueous-ethanolic phase. To confirm salt formation, sodium gliclazide was fully characterized by spectroscopy, differential scanning calorimetry, and potentiometric titration. Furthermore, solubility and in vitro dissolution studies of formulated tablets were performed at $\mathrm{pH}$ values of $1.2,4.5$, and 6.8 . Sodium gliclazide demonstrated a significant increase in solubility at $\mathrm{pH}$ values of 4.5 and 6.8. The most apparent increase was achieved in unbuffered distilled water with a 235-fold higher solubility. Moreover, sodium gliclazide showed an enhancement in the dissolution rate in all tested media, but most significantly at $\mathrm{pH} 4.5$ and 6.8. The highest difference (60\%) in dissolution rate between gliclazide and its sodium salt was obtained at $\mathrm{pH} 6.8$ at $30 \mathrm{~min}$.

The sodium salt of gliclazide presents improved solubility and drug dissolution, therefore limiting the possibility of variable absorption and improving the onset of action with potential enhancement in its overall bioavailability.
\end{abstract}

KEYWORDS: Solubility; dissolution; sodium gliclazide; antidiabetic; onset.

\section{INTRODUCTION}

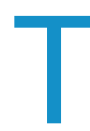

he rate of dissolution of active ingredients exhibiting poor aqueous solubility is a fundamental determinant of rate of absorption, hence oral bioavailability (1). According to the Biopharmaceutics Classification System (BCS), the dissolution of drugs demonstrating low solubility-high permeability (Class II) may be considered as the rate-limiting step through which possible in vivo behavior may be anticipated in terms of onset of action and intensity of pharmacological effect $(2,3)$. It is therefore recommended to conduct the in vitro assessment of dissolution of Class II drugs in multiple media as an indicative test of their in vivo effect (4).

Gliclazide (GLZ) is an oral hypoglycemic sulfonylurea derivative (Figure 1) that is commonly used for the treatment of noninsulin dependent diabetes mellitus. Being a Class II drug with low aqueous solubility (5-7), GLZ exhibits an unpredictable and slow absorption rate that may in turn reflect considerable intra- and intersubject variability (8). Various attempts aimed at improving solubility and dissolution rate of GLZ have been reported. One approach involves the preparation of solid dispersions of GLZ with hydrophilic carriers such as polyethylene glycols through applying different methodologies including fusion techniques (9-12), cogrinding methods (13), solvent melting, and solvent evaporation methods (14).

Another approach that has been investigated extensively is the complexation of poorly water-soluble drugs with cyclodextrins, which was found to improve the aqueous

${ }^{*}$ Corresponding author. solubility of such drugs (15-19). Enhanced GLZ dissolution was also achieved via formulation of ordered mixtures of the hydrophobic drug with water-soluble carriers of larger particle size such as mannitol and lactose (7). Cationic and anionic surfactant micelles have also been studied as solubility enhancers for GLZ (5).

Oral absorption of GLZ is accelerated when the drug is suspended in polyethylene glycol 400 and contained in a soft gelatin capsule (20). On the other hand, floating alginate beads utilizing biodegradable polymers are able to maintain reduced blood glucose levels as a consequence of improved systemic absorption of GLZ (21). The solubility of GLZ increased significantly with $\mathrm{pH}$ modification of the medium (water and phosphate buffers at different $\mathrm{pH}$ values) combined with the use of different cosolvents (22).

In another study (23), the in situ micronization of GLZ through recrystallization in the presence of various stabilizers achieved a desired morphology of crystals that exhibited a faster dissolution rate. In situ micronization techniques produce more thermodynamically stable micron-sized particles than conventional high energy milling procedures, which was found to be advantageous for certain formulation aspects $(6,23)$.

These studies suggest various mechanisms through which the solubility of GLZ is increased. These mechanisms may involve decreased aggregation of hydrophobic particles and therefore increased wettability and dispersability, decreased particle size, limited particle surface energy variations, a change in crystal habit, and conversion from crystalline to amorphous state. 


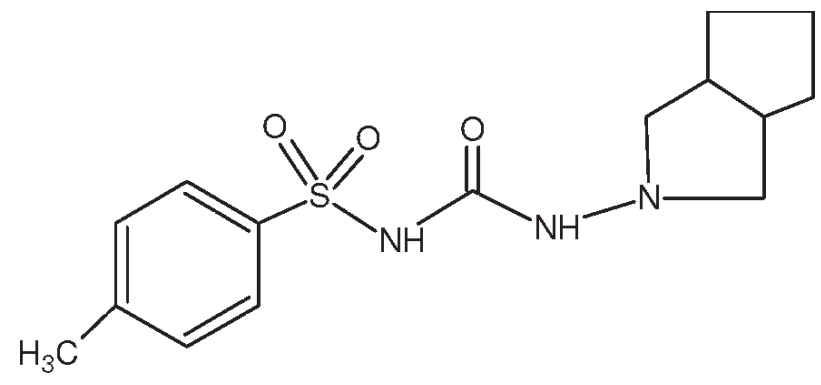

Figure 1. Chemical structure of gliclazide.

Another well-accepted approach for improving the solubility and dissolution rate of low aqueous solubility drugs is to prepare a suitable salt form of the drug $(24,25)$. However, to the best of our knowledge, there has been no salt form reported for GLZ. In this study we describe the preparation, characterization, and in vitro evaluation of a sodium salt of GLZ (Na-GLZ).

\section{MATERIALS AND METHODS \\ Materials}

GLZ was a gift from Pharma International (Amman, Jordan). Potassium dihydrogen phosphate was obtained from Sigma-Aldrich (Germany). HPLC grade acetonitrile and ethanol were obtained from Tedia Company (Tedia Inc., USA). Sodium hydroxide was obtained from Gainland Chemical Company (UK).

\section{Preparation of $\mathrm{Na}-\mathrm{GLZ}$}

Nine grams of powdered GLZ were dissolved in a solution composed of $700 \mathrm{~mL}$ ethanol and $200 \mathrm{~mL}$ distilled water. An equimolar amount of sodium hydroxide (as $1 \mathrm{M}$ solution) was added to the solution and mixed well. Immediately a very fine precipitate appeared, and the mixture was left on standing for $30 \mathrm{~min}$. The precipitated material was filtered, left to dry for $2 \mathrm{~h}$ in a fume cupboard, and then placed in a desiccator for $48 \mathrm{~h}$.

\section{Differential Scanning Calorimetry (DSC)}

Untreated GLZ and Na-GLZ were subjected to differential scanning calorimetric analyses using a Mettler Toledo calorimeter (Mettler, Toledo DSC823e, Switzerland) configured to a Mettler Star software system (Mettler, Toledo, Switzerland). Powder samples (4-5 mg) were weighed and scanned in sealed $40-\mu \mathrm{L}$ aluminium pans with pierced covers. The instrument was calibrated with indium as a reference. Thermograms were recorded under dry nitrogen atmosphere $(80 \mathrm{~mL} / \mathrm{min})$ over a $30-350^{\circ} \mathrm{C}$ temperature range and at a heating rate of $10^{\circ} \mathrm{C} / \mathrm{min}$.

\section{Spectroscopic Characterization}

Fourier transform infrared (FTIR) spectra were recorded using a Shimadzu FTIR spectrometer (Shimadzu 8400 S IR spectrophotometer, Japan) for untreated GLZ and Na-
GLZ samples prepared according to the $\mathrm{KBr}$ disk method. IR spectra were determined between 500 and $4000 \mathrm{~cm}^{-1}$. ${ }^{1} \mathrm{H}$ nuclear magnetic resonance (NMR) spectra were recorded for GLZ and Na-GLZ samples using a $350 \mathrm{MHz}$ Bruker NMR spectrometer. Furthermore, UV characterization was conducted of GLZ and Na-GLZ solutions at 25 $\mu \mathrm{g} / \mathrm{mL}$. Absorption spectra were recorded in the range of 200-350 nm.

\section{Potentiometric Titration of $\mathrm{Na}-\mathrm{GLZ}$}

A sample of solid $\mathrm{Na}-\mathrm{GLZ}(150 \mathrm{mg})$ was dissolved in 30 $\mathrm{mL}$ of distilled water and titrated potentiometrically with standardized $0.1 \mathrm{M} \mathrm{HCl}$ against $200 \mathrm{mg}$ of sodium carbonate. $\mathrm{HCl}$ was added in increments of $0.5 \mathrm{~mL}$ until the $\mathrm{pH}$ became almost constant for five successive readings. The end point was determined from the maximum point in the first derivative plot of the titration curve.

\section{Solubility Studies}

Solubility studies were conducted in an attempt to determine the saturation solubility of untreated GLZ and $\mathrm{Na}-\mathrm{GLZ}$ in different media: $\mathrm{pH} 1.2,4.5$, and 6.8 in addition to distilled water. Gradual addition of untreated GLZ or $\mathrm{Na}-\mathrm{GLZ}$ to glass vials containing $1 \mathrm{~mL}$ of each medium was carried out until the solid added no longer dissolved and a precipitate was clearly present. The glass vials were placed in a shaker water bath at $37 \pm 0.1^{\circ} \mathrm{C}$ for $48 \mathrm{~h}$ to reach equilibrium. Subsequently, the contents were filtered through $0.45-\mu \mathrm{m}$ syringe filters. A volume of $100 \mu \mathrm{L}$ was withdrawn from the filtrate, suitably diluted, and analyzed by high performance liquid chromatography (HPLC).

The HPLC system comprised a UV detector (Merck-Hitachi, model L-7400, Tokyo-Japan), a pump (Merck-Hitachi, model L-7400, Tokyo-Japan), and an integrator unit (Merck-Hitachi, model D-7500, Tokyo-Japan). The chromatographic conditions were based on a previously published method (26). In brief, a reversed phase C18 column was employed ( $5 \mu \mathrm{m}, 200 \times 4.6 \mathrm{~mm}$ i.d., Thermo Scientific, USA). The mobile phase consisted of a mixture containing $40 \%$ acetonitrile and $60 \%$ of $25 \mathrm{mM}$ phosphate buffer at $\mathrm{pH} 3.5$ and was run at a flow rate of $2 \mathrm{~mL} / \mathrm{min}$. The monitoring wavelength was set at $235 \mathrm{~nm}$.

\section{Partition Coefficient $(\log P)$ Determination}

Octanol/water partition coefficient was determined as $\log \left(c_{\text {octanol }} / c_{\text {water }}\right)$ for both untreated GLZ and Na-GLZ. Untreated GLZ or Na-GLZ (20 mg) was added to glass tubes containing $5 \mathrm{~mL}$ of octanol and $5 \mathrm{~mL}$ of distilled water. The tightly closed tubes were placed in a shaker water bath at $37 \pm 0.1^{\circ} \mathrm{C}$ for $24 \mathrm{~h}$. The concentrations of untreated GLZ or Na-GLZ were determined in the aqueous phase using the HPLC analysis described previously in the solubility studies. Partition coefficient experiments were conducted in triplicate. 


\section{Formulation and Preparation of Tablets}

Untreated GLZ (80 mg) or an equivalent amount of $\mathrm{Na}-$ GLZ was mixed with lactose $(50 \% \mathrm{w} / \mathrm{w})$ and starch $(10 \%$ $\mathrm{w} / \mathrm{w}$ ) to obtain a final powder weight of $200 \mathrm{mg}$ for each tablet. At the concentration employed, starch attained the desired disintegration properties. Powder compression was carried out in a 7-mm die at a force of $10 \mathrm{kN}$ using a manual hydraulic press. To prevent sticking of compressed tablets, the punch and die were first lubricated with a solution of $5 \% \mathrm{w} / \mathrm{v}$ magnesium stearate in $96 \% \mathrm{v} / \mathrm{v}$ ethanol.

\section{Dissolution Tests}

Comparative dissolution experiments of untreated GLZ tablets and $\mathrm{Na}-\mathrm{GLZ}$ tablets were carried out using a Copley Scientific dissolution apparatus (DIS6000, Copley, UK). The tests were performed according to pharmacopoeial specifications using Apparatus 2 (paddle method). The three media employed for testing were $0.1 \mathrm{M} \mathrm{HCl}, \mathrm{pH} 4.5$ phosphate buffer, and pH 6.8 phosphate buffer. Paddle rotation was set at $75 \mathrm{rpm}$. Medium temperature was set at $37 \pm 0.5^{\circ} \mathrm{C}$. Six tablets of each formulation were placed one in each vessel containing $900 \mathrm{~mL}$ of the test medium. Samples $(2 \mathrm{~mL})$ were withdrawn at predetermined time points $(10,20,30,45,60$, and $120 \mathrm{~min})$, and the volume withdrawn was taken into consideration when calculating the percentage release of GLZ or $\mathrm{Na}-\mathrm{GLZ}$ in the remaining volume of test medium. Filtration of samples was performed in situ via resident probes to which polyethylene filters were connected and designed to be left in the dissolution vessel for the duration of the test.

The percentage release of gliclazide from both formulations was determined using the HPLC method described above. The linearity of the method over the expected concentration range was validated by injecting standard solutions of gliclazide in the concentration range of $18-115 \mu \mathrm{g} / \mathrm{mL}$, which covers $20-125 \%$ of the anticipated $100 \%$ concentration (i.e., the concentration resulting from the dissolution of an $80 \mathrm{mg}$ gliclazide tablet in $900 \mathrm{~mL}$ of medium). A representative calibration equation is given by: $A=23693 x-106.7$ with an average correlation coefficient of 0.9984 .

\section{RESULTS AND DISCUSSION Characterization of $\mathrm{Na}-\mathrm{GLZ}$}

The dried precipitate of $\mathrm{Na}-\mathrm{GLZ}$ was subjected to DSC analysis, and the thermogram (Figure 2) clearly shows a melting transition at $308^{\circ} \mathrm{C}$ that was also confirmed by simple measurements of the melting point of the compound. Under the same conditions, a DSC thermogram was also obtained for an untreated GLZ sample and shows a melting point at $168^{\circ} \mathrm{C}$, which is consistent with the reported values (27). In comparison, Na-GLZ exhibited a melting point that is significantly higher than that of untreated GLZ, which is consistent with the ionic nature of the salt where intermolecular attraction forces are stronger.

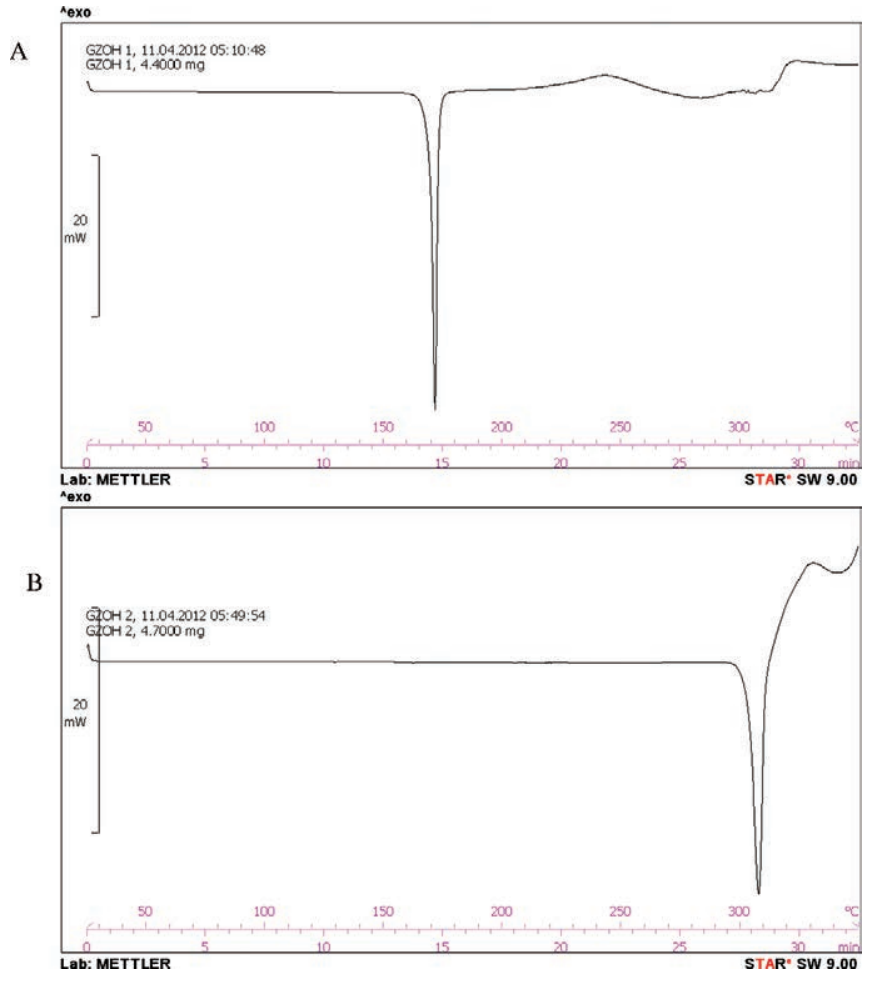

Figure 2. DSC thermograms of (A) GLZ and (B) Na-GLZ.

To ensure the absence of sodium hydroxide from the obtained solid salt, it was titrated potentiometrically with standardized $\mathrm{HCl}$ solution. Accordingly, the starting $\mathrm{pH}$ of the salt solution ( $150 \mathrm{mg} / 30 \mathrm{~mL}$ water) was 6.85 , which indicated absence of excess sodium hydroxide. Furthermore, the percentage purity of the salt on molar basis of $\mathrm{Na}-\mathrm{GLZ}$ was $98.9 \%$.

The IR spectra for untreated GLZ were consistent with those reported (14). The most significant changes in the IR spectrum of GLZ compared with that of $\mathrm{Na}-\mathrm{GLZ}$ (Figure 3) were (1) the obvious shift in the sulfonamide band from $1650 \mathrm{~cm}^{-1}$ in untreated GLZ to $1710 \mathrm{~cm}^{-1}$ in Na-GLZ and (2) the bands in the region of $3500 \mathrm{~cm}^{-1}$ that were sharper and fewer in number in the case of GLZ. These changes agree with the decreased ability to form intermolecular hydrogen bonds (involving sulfonamide oxygen and a proton) in the salt form due to loss of acidic hydrogen, while the likelihood of sulfonamide-water hydrogen bonding is increased in the salt form. Further evidence for salt formation came from NMR spectra of the prepared salt and untreated GLZ. The broad signal at $\delta=9.9$ in the spectrum of GLZ, which corresponds to the acidic sulfonamide proton, is completely absent in the spectrum of $\mathrm{Na}-\mathrm{GLZ}$ indicating the replacement of hydrogen by sodium.

UV spectra for solutions prepared to contain the same mass of either GLZ or Na-GLZ were almost identical, with $\lambda_{\max }$ at $233 \mathrm{~nm}$. The observation that the Na-GLZ spectrum showed slightly less absorbance (93.3\%) than that of GLZ over the entire wavelength range is attributed to the content of sodium in the prepared salt, which accounts 
A

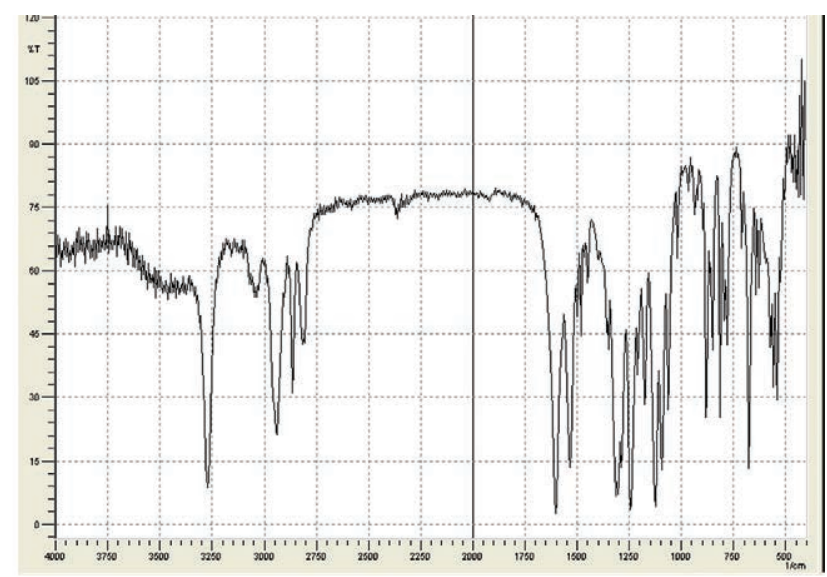

B

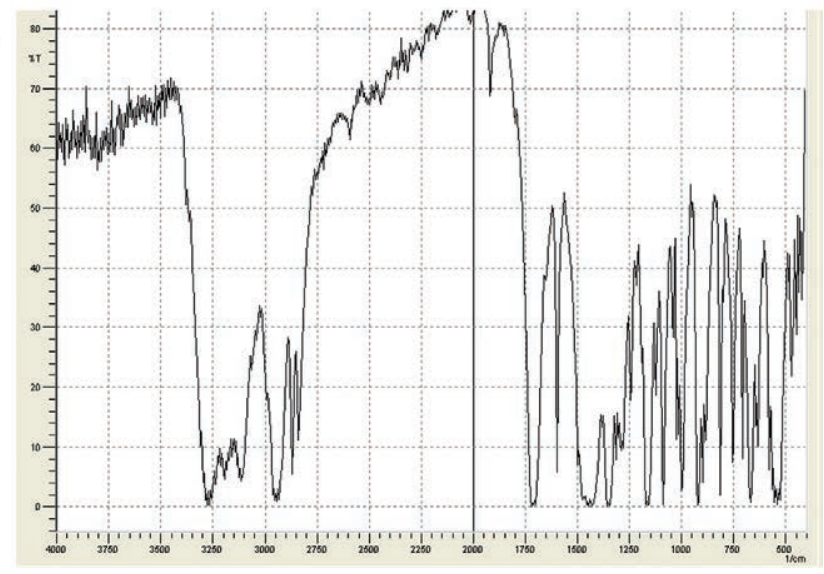

Figure 3. FTIR spectra of (A) GLZ and (B) Na-GLZ.

for almost the same percentage (6.64\%) of sodium in the molar mass of $\mathrm{Na}-\mathrm{GLZ}$.

Using a previously published stability indicating HPLC method (26), both GLZ and Na-GLZ eluted at the same retention time with no significant additional peaks. Based on a calibration curve in which standard solutions were prepared from standard GLZ, the purity of prepared $\mathrm{Na}-\mathrm{GLZ}$ was estimated to be $99.1 \%$ on a molar basis. The purity calculated on a mass basis for GLZ salt was $92.1 \%$, which again accounts for the percentage of sodium and the estimated water content $(0.14 \%$ determined by Karl Fisher method) in Na-GLZ. Thus the identity and purity of the prepared material was confirmed to be the sodium salt of GLZ with a purity of $99.1 \%$.

\section{Solubility and Partition Coefficient}

The solubility of GLZ as well as prepared Na-GLZ was determined in distilled water and in media of three relevant $\mathrm{pH}$ values $(1.2,4.5$, and 6.8$)$, as these $\mathrm{pH}$ values are generally recommended for testing the dissolution performance of solid oral dosage forms (4). A summary of the obtained solubility data is shown in Table 1.

According to the data presented in Table 1, the solubility of GLZ was $\mathrm{pH}$ dependent with a minimum solubil- ity obtained at $\mathrm{pH} 4.5$ within the examined values; the solubility increased at the extremely low pH of 1.2 , and the maximum solubility was observed in a buffer of $\mathrm{pH} 6.8$. This trend of GLZ solubility was consistent with previously published reports (28) and can be explained as GLZ is both an acid due to its sulfonamide proton with $p K_{\mathrm{a}}$ of $5.8(28$, 29) and a base due to the alicyclic aliphatic amino group with $\mathrm{p} K_{\mathrm{a}}$ of 2.9 (28). Thus, within the studied $\mathrm{pH}$ values, the drug is expected to be in its minimum ionization state at $\mathrm{pH}$ 4.5. At higher $\mathrm{pH}$ values, the sulfonamide group starts to deprotonate, acquiring a negative charge. At lower $\mathrm{pH}$ values (i.e., 1.2), the alicyclic amino group would be protonated so that the molecule would possess a positive charge. In comparison, the solubility of Na-GLZ was 17 times higher than that of GLZ in a medium of pH 4.5, 30 times higher in a medium of $\mathrm{pH} 6.8$, and 235 times higher in unbuffered distilled water. Therefore, salt formation resulted in a dramatic increase in the solubility of gliclazide, which could provide a faster dissolution rate of the drug (in distilled water, $\mathrm{pH} 4.5$, and $\mathrm{pH} 6.8$ ) and consequently a more rapid therapeutic effect.

However, at a pH value of 1.2, the solubility of the salt was even slightly less than that of GLZ, which corresponds with previous observations on GLZ itself (28), that is, at the low $\mathrm{pH}$ value (1.2), the ionization of the weakly basic amino group seems to predominate the effect of the acidic sulfonamide group that is essentially not ionized at pH 1.2. Consequently, the molecule would be slightly more soluble as a result of the polarization imposed by the positive charge on the amino nitrogen.

The octanol-water partition coefficient expressed as $\log P$ was also determined for both GLZ and $\mathrm{Na}-\mathrm{GLZ}$. The $\log P$ values of 2.04 and 0.68 were obtained for GLZ and $\mathrm{Na}-\mathrm{GLZ}$, respectively (RSD less than $3.1 \%$ ). These results agree with the previously published data for the partition coefficient of gliclazide $(7,30)$. Accordingly, the salt form is approximately 20 times more hydrophilic than the untreated form. Nevertheless, the values of $\log P$ for $\mathrm{Na}-$ GLZ are still within the recommended optimum range of typical drugs (31).

\section{Dissolution Properties of Na-GLZ}

Tablets of each of GLZ and Na-GLZ were prepared to contain the equivalent of $80 \mathrm{mg} \mathrm{GLZ}$. Content uniformity testing was performed to ensure homogeneity of the tablet mix and uniform content of the desired dose utilizing the HPLC method described in the Experimental section. The assayed percentage per label for GLZ and $\mathrm{Na}-\mathrm{GLZ}$ tablets where in both cases within $\pm 5 \%$ and with RSD values less than $1.9 \%$. Dissolution profiles were obtained for GLZ and Na-GLZ in $0.1 \mathrm{M} \mathrm{HCl}(\mathrm{pH} 1.2), \mathrm{pH} 4.5$ phosphate buffer, and $\mathrm{pH} 6.8$ phosphate buffer (4). A summary of the dissolution profiles obtained is presented in Figure 4. In all media, the dissolution rate of $\mathrm{Na}-\mathrm{GLZ}$ was higher than that of GLZ with the lowest observed difference in dissolution rate observed at $\mathrm{pH}$ 1.2. The highest difference in 
Table 1. Solubility Data for GLZ and Na-GLZ at $37 \pm 0.1{ }^{\circ} \mathrm{C}$

\begin{tabular}{|c|c|c|c|c|}
\hline & \multicolumn{4}{|c|}{ Solubility $\mu \mathrm{g} / \mathrm{mL}$ (RSD) } \\
\hline & Distilled water & 0.1 M HCl pH 1.2 & Phosphate buffer pH 4.5 & Phosphate buffer pH 6.8 \\
\hline GLZ & $52.6(4.6)$ & $124.2(2.1)$ & $40.4(3.8)$ & $182.4(5.3)$ \\
\hline $\mathrm{Na}-\mathrm{GLZ}$ & $12369.7(0.25)$ & $94.6(15.7)$ & $714(4.2)$ & $5421.7(8.3)$ \\
\hline
\end{tabular}

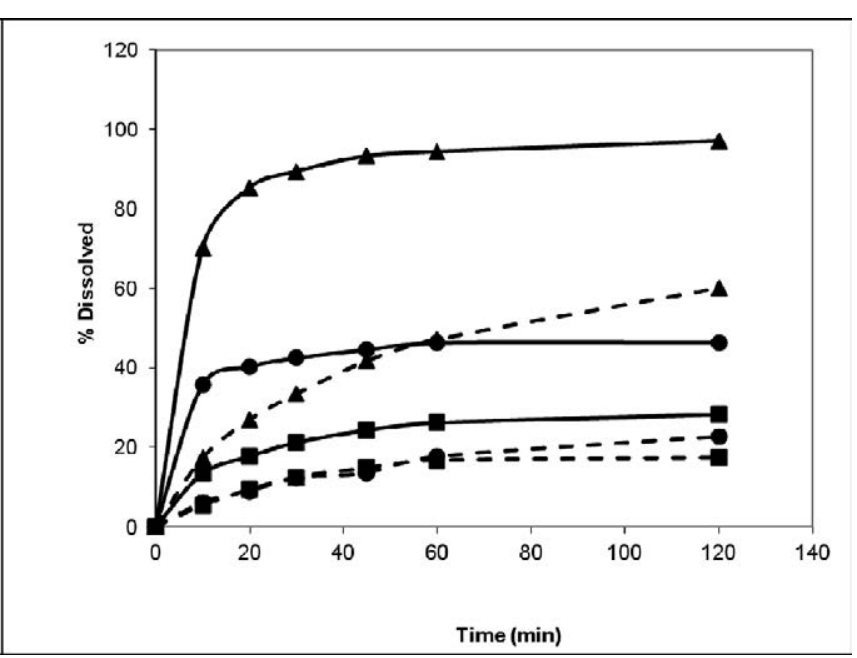

Figure 4. Dissolution profiles of gliclazide (dashed lines) and sodium gli-

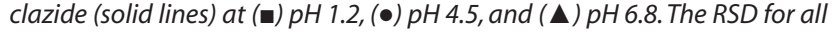
data points were generally less than $8 \%$.

dissolution rate was observed at $\mathrm{pH} 6.8$ where it reached approximately $60 \%$ at $30 \mathrm{~min}$. In the buffer medium of $\mathrm{pH}$ 4.5 , the percentage dissolved of the salt was $30 \%$ higher than that of GLZ within the first 20 min.

Nevertheless, the dissolution profile of Na-GLZ at pH 4.5 show a plateau at only $40 \%$ of drug dissolved, which was not expected in light of the solubility data of the salt at $\mathrm{pH} 4.5$ (i.e., $714 \mu \mathrm{g} / \mathrm{mL}$ ), whereas the expected $100 \%$ dissolved was $88.9 \mu \mathrm{g} / \mathrm{mL}$. In addition to the possibility of the salt being converted (to some extent) to the undissociated acid form, other reasons might contribute to the observed effect. Such reasons may include the mechanical shaking of the solubility vial versus the rotation speed of the dissolution system paddle, but the most important reason was perhaps the fact that the dissolution test was performed on formulated tablets, whereas the solubility was performed on a drug powder. One possible factor, in this regard, may be the lubricant magnesium stearate where its use in tablets is well known to affect disintegration and dissolution properties. However, the extent of dissolution for the prepared sodium salt was still at least six times higher than that achieved by untreated gliclazide at $\mathrm{pH}$ 4.5. Even at $\mathrm{pH} 1.2$ where the improvement in dissolution was the slightest, the percentage dissolved of the salt form was still double that of untreated gliclazide. Since
$\mathrm{pH} 1.2$ is almost $4 \mathrm{pH}$ units less than the $\mathrm{p} K_{\mathrm{a}}$ of gliclazide, the conversion of the salt to the undissociated acid form is quite likely, which explains the observed effect. Nevertheless, that should not preclude making use of the high solubility and dissolution rate demonstrated by the salt at slightly acidic and near neutral $\mathrm{pH}$ values, since this limitation could simply be overcomed by developing an enteric coated tablet formulation of the salt, which escapes the highly acidic medium of the stomach.

It is noteworthy that in each medium, the percentage release of $\mathrm{Na}-\mathrm{GLZ}$ reached its maximum within the first 15-20 min, while for GLZ it continued to increase gradually up to the end of dissolution test (120 min). This rapid dissolution observed for $\mathrm{Na}-\mathrm{GLZ}$ together with the higher overall percentage release is particularly important for a drug like gliclazide (antidiabetic) where the action is usually required to start rapidly. Clinically used formulations of GLZ tablets typically require $2-8 \mathrm{~h}$ to reach maximum plasma concentration, which could be considered a shortcoming (8). This latency in achieving maximum concentration is a result of the low dissolution rate of the drug, where a faster dissolving soft gelatin formulation was shown to achieve higher $C_{\max }$ within a $36 \%$ shorter time (20). Previous reports $(20,28,32)$ demonstrate that dissolution of gliclazide, particularly at lower $\mathrm{pH}$ values, is an important determinant of its rate of absorption and consequently its onset of action with reasonable correlation between in vitro dissolution and in vivo bioavailability. Thus, the prepared Na-GLZ might offer a potential improvement in the onset of action of gliclazide and a decrease in inter-individual variability of its absorption, which might lead to better clinical outcomes. Although several approaches have been described to improve the solubility and dissolution of gliclazide, salt formation offers the advantages of simplicity, low cost, and possibility of large-scale production.

\section{CONCLUSION}

The sodium salt of gliclazide was prepared by an easy and potentially large-scale method. The prepared salt was fully characterized using DSC, HPLC, NMR, UV, and IR methods. Solubility of the salt was determined in different media and found to increase several-fold in comparison to gliclazide. The dissolution rates of sodium gliclazide in the investigated media were also favorable demonstrating significantly faster dissolution. The most significant 
improvement in the dissolution rate was obtained at the intestinal $\mathrm{pH}$ of 6.8. For a hypoglycemic drug such as gliclazide where a rapid pharmacological effect is usually sought, the observed improvement in the dissolution rate may result in an enhanced clinical outcome for patients who use the drug.

\section{ACKNOWLEDGMENT}

The authors wish to thank the Deanship of Academic Research at The University of Jordan for financial support (Grant recommendation No. 3/2009-2010).

\section{REFERENCES}

1. Dressman, J. B.; Amidon, G. L.; Reppas, C.; Shah, V.P. Dissolution Testing as a Prognostic Tool for Oral Drug Absorption: Immediate Release Dosage Forms. Pharm. Res. 1998, 15 (1), 11-22. DOI: 10.1023/A:1011984216775.

2. Amidon, G. L.; Lennernäs, H.; Shah, V. P.; Crison, J. R. A Theoretical Basis for a Biopharmaceutic Drug Classification: The Correlation of in Vitro Drug Product Dissolution and in Vivo Bioavailability. Pharm. Res. 1995, 12 (3), 413-420. DOI: 10.1023/A:1016212804288.

3. Löbenberg, R.; Amidon, G.L. Modern bioavailability, bioequivalence and biopharmaceutics classification system. New scientific approach to international regulatory standards. Eur. J. Pharm. Biopharm. 2000, 50 (1), 3-12. DOI: 10.1016/S0939-6411(00)00091-6.

4. Dissolution Testing of Immediate Release Solid Oral Dosage Forms; Guidance for Industry; U.S. Department of Health and Human Services, Food and Drug Administration, Center for Drug Evaluation and Research (CDER), U.S. Government Printing Office: Washington, DC, 1997.

5. Alkhamis, K. A.; Allaboun, H.; Al-Momani, W. Y. Study of the solubilization of gliclazide by aqueous micellar solutions. J. Pharm. Sci. 2003, 92 (4), 839-846. DOI: 10.1002/jps.10350.

6. Varshosaz, J.; Talari, R.; Mostafavi, S.A.; Nokhodchi, A. Dissolution enhancement of gliclazide using in situ micronization by solvent change method. Powder Technol. 2008, 187 (3), 222-230. DOI: 10.1016/j.powtec.2008.02.018.

7. Saharan, V. A.; Choudhury, P. K. Dissolution rate enhancement of gliclazide by ordered mixing. Acta Pharm. 2011, 61 (3), 323-334. DOI: 10.2478/v10007011-0021-7.

8. Palmer, K. J.; Brogden, R. N. Gliclazide-an update of its pharmacological properties and therapeutic efficacy in non-insulin-dependent diabetes-mellitus. Drugs 1993, 46 (1), 92-125. DOI: 10.2165/00003495-19934601000007.

9. Patil, M. P.; Gaikwad, N. J. Preparation and characterization of gliclazide-polyethylene glycol 4000 solid dispersions. Acta Pharm. 2009, 59 (1), 57-65. DOI: 10.2478/v10007-009-0001-3.
10. Khattab, I. S.; Nada, A.; Zaghloul, A. A. Physicochemical characterization of gliclazide-macrogol solid dispersion and tablets based on optimized dispersion. Drug Dev. Ind. Pharm. 2010, 36 (8), 893-902. DOI: $10.3109 / 03639040903578734$.

11. Patil, M. P.; Gaikwad, N. J. Characterization of gliclazide-polyethylene glycol solid dispersion and its effect on dissolution. Braz. J. Pharm. Sci. 2011, 47 (1), 161166. DOI: 10.1590/S1984-82502011000100020.

12. Hosmani, A. H.; Thorat, Y. S. Optimization and pharmacodynamic evaluation of solid dispersion of gliclazide for dissolution rate enhancement. Lat. Am. J. Pharm. 2011, 30 (8), 1590-1595.

13. Barzegar-Jalali, M.; Valizadeh, H.; Shadbad, M. R. S.; Adibkia, K.; Mohammadi, G.; Farahani, A.; Arash, Z.; Nokhodchi, A. Cogrinding as an approach to enhance dissolution rate of a poorly water-soluble drug (gliclazide). Powder Technol. 2010, 197 (3), 150-158. DOI: 10.1016/j.powtec.2009.09.008.

14. Biswal, S.; Sahoo, J.; Murthy, P. N.; Giradkar, R. P.; Avari, J. G. Enhancement of dissolution rate of gliclazide using solid dispersions with polyethylene glycol 6000 . AAPS PharmSciTech 2008, 9 (2), 563-570. DOI: 10.1208/ s12249-008-9079-z.

15. Moyano, J. R.; Arias-Blanco, M. J.; Ginés, J. M.; Giordano, F. Solid-state characterization and dissolution characteristics of gliclazide- $\beta$-cyclodextrin inclusion complexes. Int. J. Pharm. 1997, 148 (2), 211-217. DOI: 10.1016/ S0378-5173(96)04848-X.

16. Aggarwal, S.; Singh, P. N.; Mishra, B. Studies on solubility and hypoglycemic activity of gliclazide beta-cyclodextrin-hydroxypropylmethylcellulose complexes. Pharmazie 2002, 57 (3), 191-193.

17. Sapkal, N. P.; Kilor, V. A.; Bhusari, K. P.; Daud, A. S. Evaluation of some methods for preparing gliclazide beta-cyclodextrin inclusion complexes. Trop. J. Pharm. Res. 2007, 6 (4), 833-840. DOI: 10.4314/tjpr. v6i4.14667.

18. Nacsa, 'A.; Ambrus, R.; Berkesi, O.; Szabó-Révész, P.; Aigner, Z. Water-soluble loratadine inclusion complex: analytical control of the preparation by microwave irradiation. J. Pharm. Biomed. Anal. 2008, 48 (3), 10201023. DOI: 10.1016/j.jpba.2008.07.001.

19. Salústio, P. J.; Cabral-Marques, H. M.; Costa, P. C.; Pinto, J. F. Comparison of ibuprofen release from minitablets and capsules containing ibuprofen: $\beta$-Cyclodextrin complex. Eur. J. Pharm. Biopharm. 2011, 78 (1), 58-66. DOI: 10.1016/j.ejpb.2010.12.022.

20. Hong, S. S.; Lee, S. H.; Lee, Y. J.; Chung, S. J.; Lee, M. H.; Shim, C. K. Accelerated oral absorption of gliclazide in human subjects from a soft gelatin capsule containing a PEG 400 suspension of gliclazide. J. Controlled Release 1998, 51 (2-3), 185-192. DOI: 10.1016/S01683659(97)00167-3.

21. Awasthi, R.; Kulkarni, G. T. Development of novel gastroretentive floating particulate drug delivery system 
of gliclazide. Curr. Drug Delivery 2012, 9 (5), 437-451. DOI: 10.2174/156720112802650716.

22. Seedher, N.; Kanojia, M. Co-solvent solubilization of some poorly-soluble antidiabetic drugs. Pharm. Dev. Technol. 2009, 14 (2), 185-192. DOI: $0.1080 / 10837450802498894$.

23. Talari, R.; Varshosaz, J.; Mostafavi, S. A.; Nokhodchi, A. Dissolution enhancement of gliclazide using $\mathrm{pH}$ change approach in presence of twelve stabilizers with various physico-chemical properties. J. Pharm. Pharm. Sci. 2009, 12 (3), 250-265.

24. Bani-Jaber, A.; Hamdan, I.; Al-Khalidi, B. Sodium mefenamate as a solution for the formulation and dissolution problems of mefenamic acid. Chem. Pharm. Bull. 2007, 55 (8), 1136-1140. DOI: 10.1248/ cpb.55.1136.

25. Hosmani, A. H.; Thorat, Y. S. Synthesis and evaluation of nanostructured particles of salt of ketoconazole for solubility enhancement. Dig. J. Nanomater. Bios. 2011, $6(3), 1411-1418$.

26. Bansal, G.; Singh, M.; Jindal, K. C. Forced degradation study on gliclazide and application of validated stability-indicating HPLC-UV method in stability testing of gliclazide tablets. Chromatographia 2007, 66 (9-10), 751-755. DOI: 10.1365/s10337-007-0394-4.

27. Moffat, A. C.; Jackson, J. V.; Moss, M. S.; Widdop, B. Analytical and Toxicological Data Monographs (Gliclazide). In Clarke's Isolation and Identification of Drugs in Pharmaceuticals, Body Fluids, and Post-mortem
Material. 2nd ed; Clarke, E. G. C., Moffat, A. C., Eds.; The Pharmaceutical Press: London, 1986; p 640.

28. Grbic, S.; Parojcic, J.; Ibric, S.; Djuric, Z. In vitro-in vivo correlation for gliclazide immediate-release tablets based on mechanistic absorption simulation. AAPS PharmSciTech 2011, 12 (1), 165-171. DOI: 10.1208/ s12249-010-9573-y.

29. Narasimham, L.; Barhate, V. D. Physico-chemical characterization of some beta blockers and antidiabetic drugs - potentiometric and spectrophotometric pKa determination in different co-solvents. Eur. J. Chem. 2011, 2 (1), 36-46. DOI: 10.5155/eurjchem.2.1.36-46.371.

30. Wassvik, C. M.; Holmén, A. G.; Bergström, C. A. S.; Zamora, I.; Artursson, P. Contribution of solid-state properties to the aqueous solubility of drugs. Eur. J. Pharm. Sci. 2006, 29 (3-4), 294-305. DOI: 10.1016/j. ejps.2006.05.013.

31. Lipinski, C. A.; Lombardo, F.; Dominy, B. W.; Feeney, P. J. Experimental and computational approaches to estimate solubility and permeability in drug discovery and development settings. Adv. Drug Delivery Rev. 1997, 23 (1-3), 3-25. DOI: 10.1016/S0169409X(96)00423-1.

32. Al-Kassas, R. S.; Al-Gohary, O. M. N.; Al-Faadhel, M. $\mathrm{M}$. Controlling of systemic absorption of gliclazide through incorporation into alginate beads. Int. J. Pharm. 2007, 341 (1-2), 230-237. DOI: 10.1016/j. ijpharm.2007.03.047. 